\title{
Outcomes among inpatients with cirrhosis and Clostridioides difficile infection in the modern era: results from an analysis of the National Inpatient Sample
}

\author{
Sentia Iriana ${ }^{a *}$, Sachit Sharma ${ }^{b *}$, Stephanie McDonough ${ }^{a}$, Eduardo Rodriguez Zarate ${ }^{a}$, Douglas G. Adler ${ }^{a}$ \\ University of Utah, Salt Lake City, UT; University of Toledo, OH, USA
}

Abstract

Background Patients with cirrhosis are at increased risk of Clostridioides difficile infection (CDI). We analyzed outcomes and healthcare utilization in hospitalized cirrhotic patients with CDI.

Methods The Nationwide Inpatient Sample from 2016-2017 identified 8245 hospitalized patients with a concurrent diagnosis of cirrhosis and CDI. Our primary outcome was in-hospital all-cause mortality. Secondary outcomes were length of stay (LOS), hospitalization charges and costs, shock, sepsis, acute kidney injury (AKI), intensive care unit (ICU) admission, and home discharge.

Results There was no significant difference in all-cause in-hospital mortality between patients with cirrhosis compared to patients without cirrhosis (adjusted odds ratio [aOR] 1.31, 95\% confidence interval [CI] 0.89-1.93; $\mathrm{P}=0.16)$. Patients with cirrhosis had a slightly but statistically significantly longer mean LOS ( +0.57 days, $\mathrm{P}=0.001)$. The adjusted difference in mean hospitalization charges was greater in patients with cirrhosis $(\$+4094,95 \% \mathrm{CI} \$ 1080-7108 ; \mathrm{P}=0.008)$, as was the mean hospitalization cost $(\$+1349,95 \% \mathrm{CI} \$ 600-2098 ; \mathrm{P}<0.001)$. There was no difference in the likelihood of sepsis, ICU admission, or home discharge between the groups. Patients with cirrhosis were significantly less likely to develop AKI (aOR 0.82, 95\%CI 0.72-0.93; P=0.003).

Conclusions Mortality outcomes associated with CDI have improved over time. Patients with cirrhosis continue to exhibit greater LOS and hospital costs.

Keywords Cirrhosis, Clostridioides difficile infection, acute kidney injury, National Inpatient Sample

Ann Gastroenterol 2021; 34 (1): 1-7

\section{Introduction}

The United States (US) burden of Clostridioides difficile infection (CDI) is estimated at 500,000 cases annually with 15,000-30,000 US deaths, and associated acute care inpatient

aDepartment of Gastroenterology and Hepatology, University of Utah, Salt Lake City UT (Sentia Iriana, Stephanie McDounough, Eduardo Rodriguez Zarate, Douglas G. Adler); ${ }^{\mathrm{b}}$ Department of Internal Medicine, University of Toledo, Toledo OH (Sachit Sharma), USA

Conflict of Interest: None

*Sentia Iriana and Sachit Sharma are co-first authors

Correspondence to: Douglas G. Adler MD, FACG, AGAF, FASGE, Professor of Medicine, Director, GI Fellowship Program,

Gastroenterology and Hepatology, University of Utah School of

Medicine, Huntsman Cancer Center, 30N 1900E 4R118, Salt Lake City,

Utah 84132, USA, e-mail: douglas.adler@hsc.utah.edu

Received 10 November 2020; accepted 22 March 2021; published online 14 June 2021

DOI: https://doi.org/10.20524/aog.2021.0646 costs alone exceeding $\$ 4.8$ billion $[1,2]$. Primary and secondary infections have been implicated as major causes of morbidity and mortality among patients with cirrhosis $[1,2]$. Patients with cirrhosis are uniquely predisposed to develop CDI, given their frequent hospitalizations, acquired immune dysfunction, and alterations of gut microbiota with advancing disease [3-7].

Older studies of a national registry have demonstrated that CDI has been associated with significantly higher rates of mortality, longer lengths of stay (LOS) and greater hospital charges in hospitalized patients with cirrhosis $[7,8]$. While CDI has become increasingly prevalent in patients with cirrhosis over the last 2 decades, prior studies have observed that the inpatient mortality has significantly decreased over the same time period $[7,8]$. This is probably related to increasing awareness of CDI, efforts toward prevention and infection control, as well as advancements in treatment options over time $[1,2]$. Despite this, CDI continues to be associated with higher healthcare utilization among hospitalized cirrhosis patients. Healthcare costs and LOS have been so significant that a modelling study by Saab et al proposed screening of asymptomatic hospitalized cirrhotics for CDI $[4,8]$. We 
performed an analysis of the most current data available from the National Inpatient Sample (NIS) 2016-2017 to investigate clinical outcomes and healthcare utilization in hospitalized cirrhotic patients with CDI and to look for changes from outcomes reported in older studies.

\section{Materials and methods}

\section{Data source}

We queried the Healthcare Cost and Utilization Project (HCUP) NIS databases for the years 2016 and 2017. This database of inpatient stays is derived from billing data submitted by hospitals to statewide organizations, based on discharge information. The NIS 2016 database contains data from 7.1 million (unweighted) hospital stays in 4575 hospitals in 47 US states, while the NIS 2017 database contains data from 7.1 million (unweighted) hospital stays in 4584 hospitals in 48 US states. The NIS contains de-identified clinical and nonclinical elements at both the patient and hospital level, using the International Classification of Diseases, 10th revision, Clinical Modification (ICD-10-CM) coding system. This study was performed using the 2016 and 2017 NIS databases with ICD-10-CM codes to generate a comprehensive study group of patients and procedures.

\section{Study population}

We used the newest ICD-10-CM codes to identify the following: (A) patients with a primary diagnosis of CDI; and (B) patients with a secondary diagnosis of cirrhosis. Patients were excluded if they were $<18$ years old. The ICD10-CM diagnostic and procedural codes used in this study are presented in Supplementary Table 1. Fig. 1 shows the inclusion and exclusion criteria in a flow diagram. This study was deemed exempt from approval by the institutional review board of the University of Toledo and University of Utah, as it was performed using de-identified and publicly available data.

\section{Study variables}

Patient demographics collected include: age, sex, race (Caucasian, Black, Hispanic, Asian, Pacific Islander, Native American, and other), median household income (based on patient's zip code), primary expected payer (Medicare, Medicaid, private insurance, and uninsured), hospital size (i.e., number of beds: small, medium, and large), teaching status, hospital region (Northeast, Midwest, South, and West), and urban location. The comorbidity burden was assessed using the Charlson comorbidity index. Mortality rate, patient discharge, hospital LOS, total charges (the amount billed by the hospital for the rendered services), as well as the (actual) cost of care were all obtained directly from the NIS database.
The total hospitalization charge is the amount billed to the patient and is directly available in the NIS, but it is different from the hospitalization cost, which is the actual cost incurred by the hospital in treating the patient $[9,10]$. A cost-to-charge ratio is available from HCUP and was used to calculate the hospitalization cost.

\section{Study outcomes}

Patients without cirrhosis were used as control group for the analysis of outcomes. The primary outcome was in-hospital all-cause mortality. Secondary outcomes were: (a) mean LOS; (b) mean hospitalization charges and costs; (c) shock; (d) sepsis; (e) acute kidney injury (AKI); (f) intensive care unit (ICU) admission; and (g) home discharge. Discharge disposition was divided into home vs. non-home discharge (transfer to shortterm hospital/transfer to skilled nursing facility, intermediate care facility or other facility/against medical advice/disposition unknown) per NIS definition. All outcomes were defined using standard ICD-10-CM diagnostic and procedural codes, as shown in Supplementary Table 1.

\section{Statistical analysis}

Statistical analyses were performed using STATA, version 16.0 (StataCorp., College Station, Texas, US). This software facilitates analysis to produce nationally representative unbiased results, variance estimates and P-values. Weighting of patient-level observations was implemented. Multivariate regression analysis was used to adjust for potential confounders. Univariate analysis was initially performed to calculate unadjusted odds ratios (OR). A multivariate regression model was then constructed to calculate adjusted OR (aOR). Logistic regression was used for binary outcomes and linear regression was used for continuous outcomes. Proportions were compared using the chi-square test and continuous variables were compared using the Student's $t$-test. A P-value $<0.05$ was considered statistically significant for all outcomes.

\section{Results}

\section{Patient and hospital characteristics}

We identified 202,830 patients admitted with a primary diagnosis of CDI in 2016 and 2017. We excluded 5885 patients under the age of 18 years. A total of 196,945 adult patients with CDI were included in the final analysis. Mean age was 65.95 years, and most patients were female (64.64\%). Medicare was the primary payer $(66.54 \%)$, followed by private insurance (19.87\%). The majority of patients were white (77.8\%). Patients were predominantly admitted to teaching hospitals (60.55\%). A total of $8245(4.18 \%)$ patients had a concurrent diagnosis of 


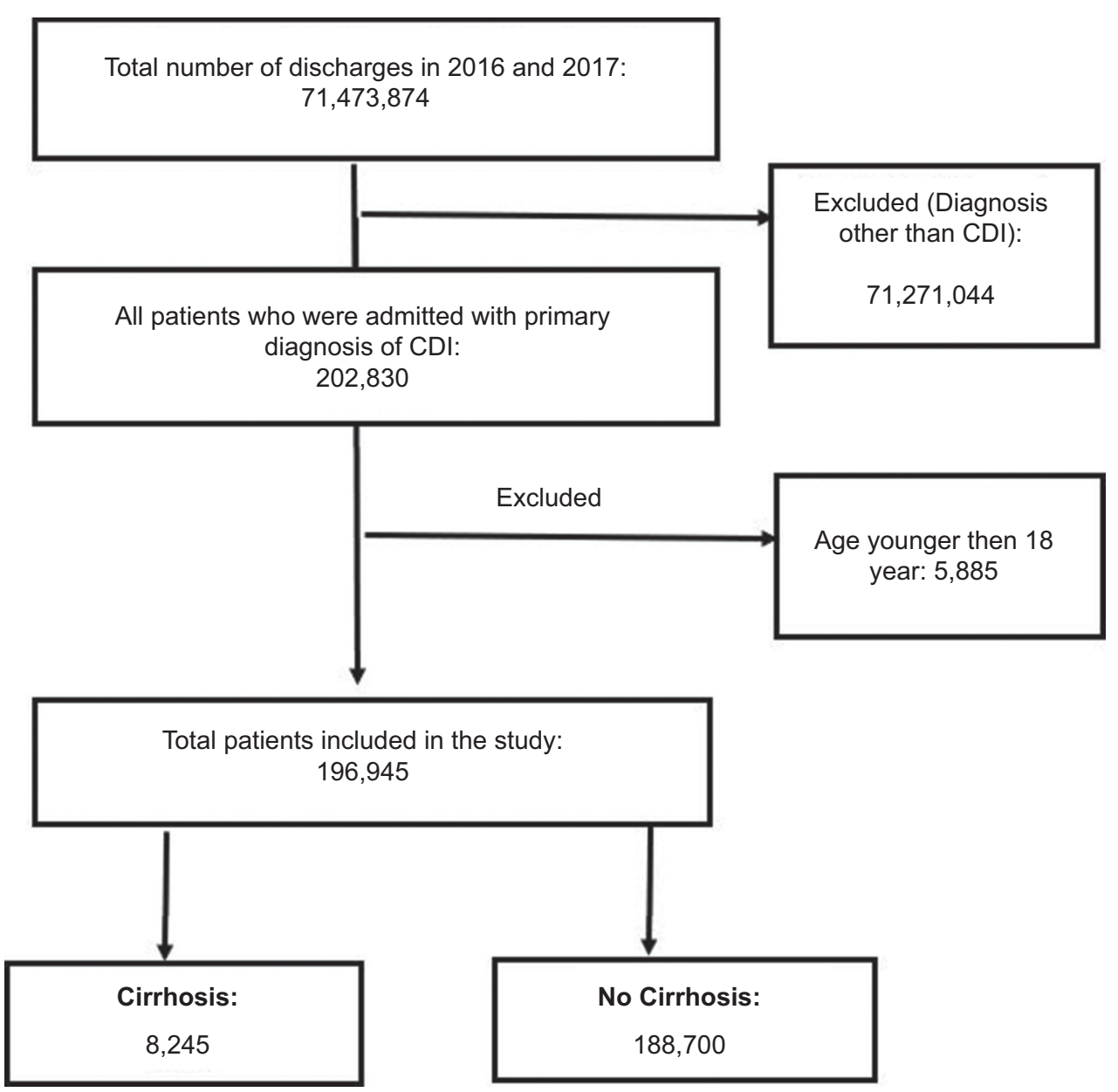

Figure 1 Flow diagram showing patient selection process CDI, Clostridioides difficile infection

cirrhosis, of whom $1.79 \%$ had portal hypertensions. Diagnostics codes including "esophageal varices," "variceal bleed," "ascites," and "portal hypertension" were used as surrogates for portal hypertension. Patients with portal hypertension had higher mortality compared to patients without portal hypertension (2.97\% vs. $1.31 \%, \mathrm{P}<0.001)$. The complete patient and hospital characteristics are presented in Tables 1 and 2. The in-hospital outcomes of patients with CDI are shown in Table 3.

\section{All-cause in-hospital mortality}

Total all-cause in-hospital mortality in patients with CDI was $1.33 \%(2635 / 196,945$ patients). The total mortality was $1.94 \%$ (580/8245 patients) in patients with cirrhosis and $1.31 \%$ $(2835 / 188,700$ patients) in patients without cirrhosis. On multivariate analysis, there was no significant difference in allcause in-hospital mortality between patients with cirrhosis and those without (aOR 1.31, 95\% confidence interval [CI] 0.89$1.93 ; \mathrm{P}=0.16)$.

\section{Mean LOS}

The overall mean LOS in patients admitted with CDI was 5.4 days. Mean LOS was 6.5 days $(95 \%$ CI 6.2-6.8) in patients with cirrhosis and 5.42 days (95\%CI 5.37-5.48) in patients without cirrhosis. On multivariate regression analysis, patients with cirrhosis had a slightly but statistically significantly longer mean LOS compared to patients without cirrhosis $(+0.57$ days, $\mathrm{P}=0.001)$.

\section{Mean hospitalization charges and costs}

The mean hospitalization charges and cost for patients admitted with CDI were $\$ 40,768$ and $\$ 10,078$, respectively. Patients with cirrhosis had higher mean hospitalization charges $(\$ 50,836$ vs. $\$ 40,367)$ and costs $(\$ 12,614$ vs. $\$ 9977)$ when compared to patients without cirrhosis. The adjusted difference in mean hospitalization charges was \$ +4094 (95\%CI \$10807108; $\mathrm{P}=0.008)$, and the difference in mean hospitalization costs was $\$+1349$ (95\%CI $\$ 600-2098 ; \mathrm{P}<0.001)$. 
Table 1 Characteristics of patients admitted to hospital with Clostridioides difficile infection

\begin{tabular}{|c|c|}
\hline Variable & $\mathrm{n}(\%)$ \\
\hline Total study population & 196,945 \\
\hline Female & $127,305(64.64 \%)$ \\
\hline Mean age (years) & 65.95 \\
\hline \multicolumn{2}{|l|}{ Insurance provider } \\
\hline Medicare & $131,047(66.54 \%)$ \\
\hline Medicaid & $22,176(11.26 \%)$ \\
\hline Private & $39,133(19.87 \%)$ \\
\hline Uninsured & $4589(2.33 \%)$ \\
\hline \multicolumn{2}{|c|}{ Charleston comorbidity index } \\
\hline 0 & $51,934(26.37 \%)$ \\
\hline 1 & $40,807(20.72 \%)$ \\
\hline 2 & $33,599(17.06 \%)$ \\
\hline 3 or more & $70,605(35.85 \%)$ \\
\hline \multicolumn{2}{|c|}{ Median income in patient zip code } \\
\hline$\$ 1-\$ 38,999$ & $54,869(27.86 \%)$ \\
\hline$\$ 39,000-\$ 47,999$ & $53,746(27.29 \%)$ \\
\hline$\$ 48,000-\$ 62,999$ & $47,818(24.28 \%)$ \\
\hline$\$ 63,000$ & $40,511(20.57 \%)$ \\
\hline \multicolumn{2}{|l|}{ Hospital Region } \\
\hline Northwest & $36,789(18.68 \%)$ \\
\hline Midwest & $47,621(24.18 \%)$ \\
\hline South & $78,010(39.61 \%)$ \\
\hline West & $34,524(17.53 \%)$ \\
\hline \multicolumn{2}{|l|}{ Hospital location } \\
\hline Rural & $22,018(11.18 \%)$ \\
\hline Urban & $174,926(88.82 \%)$ \\
\hline \multicolumn{2}{|l|}{ Hospital size } \\
\hline Small & $41,516(21.08 \%)$ \\
\hline Medium & $58,394(29.65 \%)$ \\
\hline Large & $97,015(49.27 \%)$ \\
\hline Teaching hospital & $119,250(60.55 \%)$ \\
\hline \multicolumn{2}{|l|}{ Race } \\
\hline White & $153,223(77.8 \%)$ \\
\hline Black & $21,014(10.67 \%)$ \\
\hline Hispanic & $15,243(7.74 \%)$ \\
\hline Asian or Pacific Islander & $2560(1.3 \%)$ \\
\hline Native American & $1142(0.58 \%)$ \\
\hline Other & $3763(1.91 \%)$ \\
\hline
\end{tabular}

\section{Shock}

Of the patients with CDI, $1.23 \%$ developed shock during their hospital stay. These included $2.18 \%$ of patients
Table 2 Characteristics of patients admitted to hospital with Clostridioides difficile infection: cirrhosis vs. no-cirrhosis

\begin{tabular}{lccc}
\hline Variable & \multicolumn{3}{c}{$\mathrm{n}(\%)$} \\
\cline { 2 - 4 } & $\begin{array}{ccc}\text { Cirrhosis } \\
8245\end{array}$ & $\begin{array}{c}\text { No cirrhosis } \\
188,700\end{array}$ & P-value \\
& & &
\end{tabular}

\begin{tabular}{lcc}
\hline Female & $3896(47.26 \%)$ & $123,296(65.34 \%)$ \\
$\begin{array}{l}\text { Mean age } \\
\text { (years) }\end{array}$ & 61.76 & 66.12 \\
$\begin{array}{l}\text { Insurance } \\
\text { provider }\end{array}$ & & \\
\multicolumn{1}{c}{ Medicare } & $4789(58.08 \%)$ & $126,184(66.87 \%)$ \\
Medicaid & $1815(22.02 \%)$ & $20,436(10.83 \%)$ \\
Private & $1399(16.97 \%)$ & $37,721(19.99 \%)$ \\
Uninsured & $342(2.93 \%)$ & $4,359(2.31 \%)$
\end{tabular}

Charleston

$<0.001$

comorbidity

index

\begin{tabular}{|c|c|c|c|}
\hline 0 & $0 \%$ & $51,741(27.42 \%)$ & \\
\hline 1 & $1171(14.20 \%)$ & $39,589(20.98 \%)$ & \\
\hline 2 & $1187(14.4 \%)$ & $32,381(17.16 \%)$ & \\
\hline 3 or more & 5887 (71.4\%) & 64,989 (34.44\%) & \\
\hline $\begin{array}{l}\text { Median income } \\
\text { in patient zip } \\
\text { code }\end{array}$ & & & 0.0014 \\
\hline$\$ 1-\$ 38,999$ & $2579(31.28 \%)$ & $52,308(27.72 \%)$ & \\
\hline $\begin{array}{l}\$ 39,000- \\
\$ 47,999\end{array}$ & $2233(27.09 \%)$ & $51,515(27.3 \%)$ & \\
\hline $\begin{array}{l}\$ 48,000- \\
\$ 62,999\end{array}$ & $2033(24.66 \%)$ & $45,797(24.27 \%)$ & \\
\hline$\$ 63,000$ & $1400(16.96 \%)$ & $39,080(20.71 \%)$ & \\
\hline Hospital region & & & $<0.001$ \\
\hline Northwest & $1683(20.41 \%)$ & $35,117(18.61 \%)$ & \\
\hline Midwest & $1819(22.06 \%)$ & $45,797(24.27 \%)$ & \\
\hline South & $2973(36.06 \%)$ & $75,008(39.75 \%)$ & \\
\hline West & $1770(21.47 \%)$ & $32,778(17.38 \%)$ & \\
\hline $\begin{array}{l}\text { Hospital } \\
\text { location }\end{array}$ & & & $<0.001$ \\
\hline Rural & $594(7.2 \%)$ & $21,380(11.33 \%)$ & \\
\hline Urban & $7651(92.8 \%)$ & $167,320(88.67 \%)$ & \\
\hline Hospital size & & & 0.23 \\
\hline Small & $1744(21.15 \%)$ & $36,891(19.55 \%)$ & \\
\hline Medium & $2450(29.72 \%)$ & $52,591(27.87 \%)$ & \\
\hline Large & $4051(49.13 \%)$ & $99,218(52.58 \%)$ & \\
\hline $\begin{array}{l}\text { Teaching } \\
\text { hospital }\end{array}$ & $5658(68.63 \%)$ & $113,635(60.22 \%)$ & $<0.001$ \\
\hline Race & & & $<0.001$ \\
\hline White & $6044(73.31 \%)$ & 147,167 (77.99\%) & \\
\hline
\end{tabular}

(Contd...) 
Table 2 (Continued)

\begin{tabular}{lccc} 
Variable & \multicolumn{3}{c}{$\mathrm{n}(\%)$} \\
\cline { 2 - 3 } & Cirrhosis & $\begin{array}{c}\text { No cirrhosis } \\
188,700\end{array}$ & P-value \\
\hline Black & $765(9.28 \%)$ & $20,247(10.73 \%)$ & \\
Hispanic & $1129(13.69 \%)$ & $14,152(7.5 \%)$ & \\
Asian or & $139(1.69 \%)$ & $2,415(1.28 \%)$ & \\
Pacific & & \\
Islander & & \\
Native & $51(0.61 \%)$ & $2,040(0.58 \%)$ & \\
American & & \\
Other & $117(1.42 \%)$ & $2,679(1.42 \%)$ & \\
\hline
\end{tabular}

Table 3 In-hospital outcomes of patients admitted with Clostridioides difficile infection: cirrhosis vs. no-cirrhosis

\begin{tabular}{|c|c|c|c|}
\hline \multirow[t]{2}{*}{ Outcomes } & \multicolumn{3}{|c|}{$\begin{array}{l}\text { Study population with CDI } \\
\qquad \mathrm{n}=196,945\end{array}$} \\
\hline & $\begin{array}{c}\text { No cirrhosis } \\
188,700\end{array}$ & $\begin{array}{c}\text { Cirrhosis } \\
8,245\end{array}$ & P-value \\
\hline In-hospital mortality & $1.31 \%$ & $1.94 \%$ & \\
\hline $\mathrm{uOR}$ & Ref & 1.48 & 0.03 \\
\hline $\mathrm{aOR}$ & Ref & 1.31 & 0.16 \\
\hline $\begin{array}{l}\text { Mean length of stay } \\
\text { (days) }\end{array}$ & 5.42 & 6.55 & \\
\hline Adjusted coefficient & Ref & 0.57 & 0.001 \\
\hline Mean charge & $\$ 40,278$ & $\$ 52,015$ & \\
\hline Adjusted coefficient & Ref & $\$ 4094$ & 0.008 \\
\hline Mean cost & $\$ 9953$ & $\$ 12,960$ & \\
\hline Adjusted coefficient & Ref & $\$ 1349$ & $<0.001$ \\
\hline Shock & $1.19 \%$ & $2.18 \%$ & \\
\hline $\mathrm{uOR}$ & Ref & 1.84 & $<0.001$ \\
\hline $\mathrm{aOR}$ & Ref & 1.33 & 0.12 \\
\hline Sepsis & $0.98 \%$ & $1.09 \%$ & \\
\hline uOR & Ref & 1.11 & 0.66 \\
\hline $\mathrm{aOR}$ & Ref & 1.05 & 0.84 \\
\hline ICU admission & $0.78 \%$ & $1.51 \%$ & \\
\hline uOR & Ref & 1.94 & 0.001 \\
\hline $\mathrm{aOR}$ & Ref & 1.25 & 0.32 \\
\hline AKI & $23.29 \%$ & $24.31 \%$ & \\
\hline $\mathrm{uOR}$ & Ref & 1.05 & 0.34 \\
\hline $\mathrm{aOR}$ & Ref & 0.82 & 0.003 \\
\hline Home discharge & $57.49 \%$ & $57.97 \%$ & \\
\hline uOR & Ref & 1.02 & 0.21 \\
\hline $\mathrm{aOR}$ & Ref & 1.02 & 0.67 \\
\hline
\end{tabular}

with cirrhosis and $1.19 \%$ patients without cirrhosis. On multivariate analysis, there was no difference between the 2 groups in the likelihood of shock (aOR 1.33, 95\%CI 0.921.93; $\mathrm{P}=0.12$ ).

\section{Sepsis}

Of the patients with CDI, $0.98 \%$ had sepsis during their hospital stay. These included $1.09 \%$ of patients with cirrhosis and $0.98 \%$ of patients without cirrhosis. On multivariate analysis, there was no difference between the 2 groups in the likelihood of sepsis (aOR 1.05, 95\%CI 0.63-1.75; $\mathrm{P}=0.84$ ).

\section{AKI}

Of the patients with CDI, $23.34 \%$ had AKI during their hospital stay. These included $24.31 \%$ of patients with cirrhosis and $23.29 \%$ of patients without cirrhosis. On multivariate analysis, the patients with cirrhosis were significantly less likely to develop AKI than patients without cirrhosis (aOR 0.82, 95\%CI 0.72-0.93; $\mathrm{P}=0.003$ ).

\section{ICU admission}

Of the patients with CDI, $0.81 \%$ were admitted to the ICU during their hospital stay. These included $1.51 \%$ of patients with cirrhosis and $0.78 \%$ of patients without cirrhosis. On multivariate analysis, there was no significant difference between the 2 groups in the likelihood of ICU admission (aOR 1.25 , 95\%CI 0.79-1.97; $\mathrm{P}=0.38$ ).

\section{Home discharge}

Overall, 57.51\% patients with CDI were discharged home. These included $57.97 \%$ of the patients with cirrhosis and $57.49 \%$ of those without cirrhosis. On multivariate analysis, there was no significant difference between the groups in the likelihood of home discharge (aOR 1.02, 95\%CI 0.91-1.15; $\mathrm{P}=0.67)$.

\section{Discussion}

This study examined outcomes associated with hospitalizations of patients with cirrhosis and CDI, including LOS, all-cause in-hospital mortality and hospital charges, utilizing the de-identified NIS 2016-2017. As in the NIS analysis performed by Bajaj et al a decade ago, patients with cirrhosis and CDI were younger than those without cirrhosis (61.76 vs. $66.12, \mathrm{P}<0.001)$ and continued to exhibit greater healthcare utilization with a longer LOS and higher 
hospital charges [7]. Older data from the NIS database were further investigated by Rosenblatt et al and corroborated that CDI in hospitalized patients with cirrhosis was associated with a significantly longer hospital stay (13.7 vs. 6.9 days, $\mathrm{P}<0.001$ ) [8]. Of key importance, the overall mean LOS in patients with cirrhosis and CDI has declined from an average of 14.4 days, as noted in prior studies, to 5.4 days, possibly as a result of greater awareness of CDI, as well as new and more effective CDI treatments and improved care of advanced liver disease [8].

Patients with cirrhosis and CDI continued to have higher hospital charges compared to patients without cirrhosis $[7,8]$. Higher hospital charges are presumably related to the greater LOS among patients with cirrhosis and CDI. However, additional diagnostic testing and treatment cannot be excluded, since the difference in LOS compared to non-cirrhotic patients with CDI showed only slight statistical significance. Rosenblatt et al observed that CDI increased total charges by $20.0 \%$ (95\%CI 18.0-21.0\%; $\mathrm{P}<0.001)$ and, aside from LOS, was also the most powerful factor in predicting total charges [8]. Similar patterns have been observed with other infections occurring among cirrhotic patients: a population-based study performed by Zou et al in 2018 observed higher adjusted hospital charge among patients with cirrhosis and acute respiratory infection (ARI) compared to patients with cirrhosis but without ARI ( $\$ 122,555$ vs. $\$ 79,685$ per patient per admission; $\mathrm{P}<0.001$ ) [11].

In a study by Rosenblatt et al, which analyzed the NIS database from 1998-2014, CDI prevalence in advanced cirrhotics increased from $0.8 \%$ to $2.6 \%$, an annual percent change (APC) of $8.8 \%$ (compared to $7.6 \%$ for the general population), while CDI-related mortality decreased from $20.7 \%$ to $11.3 \%$, APC $-3.4 \%$ (compared to $-2.0 \%$ for the general population), from 1998-2014 [8]. An investigation of the NIS in 2015 observed that patients with cirrhosis and CDI had an inhospital mortality rate of $13.8 \%$, significantly greater than that of inpatients with cirrhosis alone $(8.2 \% ; \mathrm{P}<0.001)$ or $\mathrm{CDI}$ alone $(9.6 \% ; \mathrm{P}<0.001)$ [7]. Based on our new data, there appears to be no significant difference in all-cause in-hospital mortality between patients with cirrhosis and those without cirrhosis; this represents a significant change in recent years and is a key finding of our study. There was also no difference between the groups in the likelihood of sepsis, shock, ICU admission or home discharge. This may be reflective of increased vigilance, prevention and treatment over time, as rates of CDI have plateaued in the US since about 2010 [1,2].

Organ failure significantly impacts survival in patients with cirrhosis who are hospitalized with infection $[3,7,8]$. Sepsis can be a precipitating factor in acute-on-chronic liver failure, which can lead to rapidly worsening liver function in patients with cirrhosis [12]. Cirrhotics with healthcare-acquired infections have more severe liver disease, hypothesized to be secondary to an excessive imbalance in their cytokine response, leading to sepsis-related organ failures $[12,13]$. In our investigation, $1.79 \%$ of patients were identified as having portal hypertension and had higher mortality compared to patients without portal hypertension $(2.97 \%$ vs. $1.31 \%, \mathrm{P}<0.01)$. Diagnostic codes for cirrhosis and associated complications, except for ascites, have been found to have relatively high positive predictive value and accuracy within a single healthcare system. However, ICD codes for cirrhosis alone may be insufficiently sensitive, and should probably be combined with ICD codes for cirrhosis complications to better identify patients [14]. Further stratification of liver disease by model for end-stage liver disease and Child-Pugh scores was limited by the lack of laboratory data.

$\mathrm{AKI}$ is an independent prognostic risk factor for mortality among patients with CDI and without cirrhosis $[8,15,16]$. Prior studies have observed that CDI was more commonly associated with concurrent AKI (35.1\%) compared to urinary tract infections, pneumonia, spontaneous bacterial peritonitis, and cellulitis in hospitalized patients with cirrhosis. While spontaneous bacterial peritonitis has a strong association with both AKI and hepatorenal syndrome, CDI significantly increased the risk of both of these conditions $[3,7,8]$. Our study observed that while $24.31 \%$ of patients with cirrhosis and $23.29 \%$ of patients without cirrhosis had AKI, patients with cirrhosis were less likely to develop AKI than patients without cirrhosis, another key finding of this study. This could potentially be related to the earlier recognition of AKI and hence earlier intervention in patients with cirrhosis.

While using a de-identified population database such as NIS permits the study of clinical outcomes and associations across a spectrum of the nation's healthcare systems, it has inherent limitations. The NIS does not identify hospitalacquired vs. community-acquired CDI or distinguish recurrent hospitalizations, which are relevant risk factors in the transmission of infections. Inpatient observation and medical care received across multiple healthcare settings may be underrepresented $[17,18]$. Lack of granular data points precluded the assessment of severity of CDI, disease recurrence, methods of testing, timing of diagnosis, and treatment. Complications including sepsis, shock, and death are presumably related to CDI, since it has been analyzed as a primary diagnosis. However, limitations of the database preclude discriminating between direct consequence vs. sequelae of critical illness or other process. Inability to review medication lists also precludes identification of potential associated protective and risk factors, implemented treatments and changes in therapy.

While the mortality associated with CDI has declined over time, its ongoing prevalence remains impactful on healthcare utilization, including longer LOS and greater hospital costs. Interestingly, Saab et al found that screening and treating for CDI among asymptomatic inpatients with cirrhosis was cost-saving, and was associated with lower mortality compared to those who did not undergo screening [4]. Contrary to published guidelines, screening asymptomatic hospitalized cirrhotics may allow the early detection and treatment of CDI $[2,19]$. Similar screening paradigms have been used for methicillin-resistant Staphylococcus aureus infections, and for patients with inflammatory bowel disease who present with a flare [19]. Future studies are also needed to investigate the complex microbiota in patients with cirrhosis and the impact of therapies including lactulose and rifaximin used for the management of hepatic encephalopathy $[8,19,20,21]$. 
In conclusion, this study analyzed the most recent data available regarding CDI among patients with cirrhosis, using the NIS 2016-2017 to investigate clinical outcomes and healthcare utilization. Mortality outcomes associated with CDI have improved over the last decade among hospitalized patients with or without cirrhosis. Patients with cirrhosis continue to exhibit longer LOS and greater hospital costs. Prevention, early detection, advancements in treatment, and vigilant management of end-organ involvement, including AKI, are checkpoints in reducing mortality and optimizing healthcare utilization [8].

\section{Summary Box}

\section{What is already known:}

- Primary and secondary infections, including Clostridioides difficile infection (CDI) have been implicated as major causes of morbidity and mortality among patients with cirrhosis

- Organ failure significantly impacts survival in patients with cirrhosis, hospitalized with CDI

- Acute kidney injury (AKI) is an independent prognostic risk factor for mortality among patients with CDI, with or without cirrhosis

\section{What the new findings are:}

- Mortality outcomes associated with CDI have improved over the last decade among hospitalized patients with or without cirrhosis

- Patients with cirrhosis continue to exhibit longer hospital stays and greater costs

- Patients with cirrhosis were less likely to develop AKI than patients without cirrhosis

\section{References}

1. McDonald LC, Gerding DN, Johnson S, et al. Clinical practice guidelines for Clostridium difficile infection in adults and children: 2017 update by the Infectious Diseases Society of America (IDSA) and Society for Healthcare Epidemiology of America (SHEA). Clin Infect Dis 2018;66:e1-e48.

2. Dubberke ER, Olsen MA. Burden of Clostridium difficile on the healthcare system. Clin Infect Dis 2012;55 (Suppl 2):S88-S92.

3. Bajaj JS, O'Leary JG, Reddy KR, et al; NACSELD. Second infections independently increase mortality in hospitalized patients with cirrhosis: the North American consortium for the study of end-stage liver disease (NACSELD) experience. Hepatology 2012;56:2328-2335.

4. Saab S, Alper T, Sernas E, Pruthi P, Alper MA, Sundaram V. Hospitalized patients with cirrhosis should be screened for Clostridium difficile colitis. Dig Dis Sci 2015;60:3124-3129.

5. Garcia-Tsao G, Surawicz CM. Editorial: Clostridium difficile infection; yet another predictor of poor outcome in cirrhosis. Am J Gastroenterol 2010;105:114-116.

6. Acharya C, Bajaj JS. Altered microbiome in patients with cirrhosis and complications. Clin Gastroenterol Hepatol 2019;17:307-321.

7. Bajaj JS, Ananthakrishnan AN, Hafeezullah M, et al. Clostridium difficile is associated with poor outcomes in patients with cirrhosis: A national and tertiary center perspective. Am J Gastroenterol 2010;105:106-113.

8. Rosenblatt R, Mehta A, Cohen-Mekelburg S, et al. The rise of Clostridioides difficile infections and fall of associated mortality in hospitalized advanced cirrhotics. Liver Int 2019;39:1263-1270.

9. Bilal M, Tayyem O, Saraireh H, Chowdhry M, Guturu P, Abougergi MS. Upper gastrointestinal hemorrhage is associated with poor outcomes among patients with acute cholangitis: a nationwide analysis. Eur J Gastroenterol Hepatol 2019;31:586-592.

10. Arora V, Moriates C, Shah N. The challenge of understanding health care costs and charges. AMA J Ethics 2015;17:1046-1052.

11. Zou B, Yeo YH, Jeong D, et al. Higher mortality and hospital charges in patients with cirrhosis and acute respiratory illness: a population-based study. Sci Rep 2018;8:9969.

12. Gustot T, Durand F, Lebrec D, Vincent JL, Moreau R. Severe sepsis in cirrhosis. Hepatology 2009;50:2022-2033.

13. Sargenti K, Prytz H, Strand A, Nilsson E, Kalaitzakis E. Healthcareassociated and nosocomial bacterial infections in cirrhosis: predictors and impact on outcome. Liver Int 2015;35:391-400.

14. Nehra MS, Ma Y, Clark C, Amarasingham R, Rockey DC, Singal AG. Use of administrative claims data for identifying patients with cirrhosis. J Clin Gastroenterol;47:e50-e54.

15. Khanna S, Keddis MT, Noheria A, Baddour LM, Pardi DS. Acute kidney injury is an independent marker of severity in Clostridium difficile infection: a nationwide survey. J Clin Gastroenterol 2013;47:481-484.

16. Charilaou P, Devani K, John F, et al. Acute kidney injury impact on inpatient mortality in Clostridium difficile infection: A national propensity-matched study. J Gastroenterol Hepatol 2018;33:1227-1233.

17. Khera R, Krumholz HM. With great power comes great responsibility: big data research from the national inpatient sample. Circ Cardiovasc Qual Outcomes 2017;10:.

18. HCUP Methods Series. Healthcare Cost and Utilization Project (HCUP); Agency for Healthcare Research and Quality; Rockville, MD: Sep, 2016.

19. Agarwalla A, Weber A, Davey S, et al. Lactulose is associated with decreased risk of Clostridium difficile infection in decompensated cirrhosis. Clin Gastroenterol Hepatol 2017;15:953-954.

20. Kondepudi KK, Ambalam P, Nilsson I, Wadström T, Ljungh A. Prebiotic-non-digestible oligosaccharides preference of probiotic bifidobacteria and antimicrobial activity against Clostridium difficile. Anaerobe 2012;18:489-497.

21. Feuerstadt P, Hong SJ, Brandt LJ. Chronic rifaximin use in cirrhotic patients is associated with decreased rate of $C$. difficile infection. Dig Dis Sci 2020;65:632-638. 


\section{Supplementary material}

Supplementary Table 1 The International Classification of Diseases, 10th revision, (ICD-10) diagnostic and procedural codes used to generate search results

\begin{tabular}{lc}
\hline Clostridioides difficile colitis & A04.7 \\
Sepsis & R65.10, R65.11, R65.20 \\
Shock & R65.21; R57.1; R57.8; R57.9 \\
Acute kidney injury & N17.0; N17.1; N17.2; N17.8; N17.9 \\
Mechanical ventilation (A) & 5A1935Z; 5A1945Z; 5A1955Z \\
Vasopressor use (B) & 3E030XZ; 3E033XZ; 3E040XZ; 3E043XZ; 3E050XZ; 3E053XZ; 3E060XZ; 3E063XZ \\
Liver cirrhosis & K70.3; K70.30; K70.31; K74.3; K74.4; K74.5; K74.6; K74.60; K74.69; K76.6; K76.7; K76.81; K72.1; K72.10; \\
& K72.11; I85.0; I85.00; I85.01; C22.0; C22.8; K65.2; K72.10; K72.11; I86.4; I85.01; I85.11 \\
\hline Portal hypertension & $\mathrm{K} 70.31 ; \mathrm{K} 76.6 ; \mathrm{K} 76.7 ; \mathrm{K} 76.81 ; \mathrm{I} 85.0 ; \mathrm{I} 85.00 ; \mathrm{I} 85.01 ; \mathrm{I} 86.4 ; \mathrm{I} 85.01 ; \mathrm{K} 65.2$ \\
\hline
\end{tabular}

$A+B$, intensive care unit admission 